\title{
CAPITAL HUMANO Y SEGMENTACION DEL MERCADO LABORAL EN LAS ISLAS DEL ROSARIO
}

\author{
MARTHA YÁNEZ CONTRERAS* \\ MENIS MERCADO MEJIA** \\ KARINA ACEVEDO GONZÁLEZ ${ }^{* * *}$
}

Recibido 17 de Julio de 2008/Enviado para Modificación 20 de Agosto de 2008/Aceptado 24 de Septiembre de 2008

\section{RESUMEN}

Las islas del Rosario son reconocidas como uno de los mejores atractivos turísticos del mundo, sin embargo, su desarrollo en el turismo no se ha visto reflejado en una mejor calidad de vida para sus residentes, quienes mantienen altos niveles de pobreza y miseria. El presente artículo muestra una caracterización de la oferta laboral de las Islas del Rosario y estima mediante un modelo probit la probabilidad de que un residente este vinculado a un empleo en el segmento primario, que comprende los empleos con mayor estabilidad y remuneración. Los resultados muestran que el bajo nivel de educación y capacitación de los nativos les dificulta el acceso a un empleo de este tipo, no obstante, esta dificultad aumenta si es mujer, joven y/o raizal. Específicamente, una mujer con el mismo nivel de capital humano que un hombre tiene un 21,2\% menos de probabilidades de pertenecer al segmento primario, mientras que un raizal tiene un $14,8 \%$ menos de probabilidad que un no raizal.

Palabras Clave: capital humano, segmentación del mercado laboral, islas del Rosario, modelo probit, sesgo de selección.

Clasificación JEL: C25, J24, J42.

\begin{abstract}
The Rosario Island are known such as one of the better tourist attractions in the world, however, its tourist development hasn't been reflected in a better quality of life for its residents, who has high level of poverty and misery. The present article shows a characterization of the labour supply of the Rosario Island and estimates

\footnotetext{
*Facultad de Ciencias Económicas - Universidad de Cartagena. Correo Electrónico: yanezmartha@hotmail.com. ** Departamento de Investigaciones Económicas y Sociales de la Facultad de Ciencias Económicas - Universidad de Cartagena. Correo Electrónico: menis_mercado@yahoo.es.

*** Estudiante de IX semestre del programa de economía e integrante del semillero adscrito al Grupo de Investigación Mercado Laboral, de la Facultad de Ciencias Económicas - Universidad de Cartagena. Correo Electrónico:kari_k95@hotmail.com.
} 
through a probit model, the probability of a resident of belonging to the primary segment, which contains the steadiest employees. The results show that the low level of education and training of the residents hinder them the access to an employment with stability, nevertheless, this hindrance increase if the resident is a woman, young or raizal. Specifically, a women with the same level of human capital than a man has a $21,2 \%$ less of probability of obtaining a employment in the primary segment, whereas a raizal person has a $14,8 \%$ less of probability than a non raizal person.

Keywords: human capital, labor market segmentation, Rosario Islands, probit model, selection bias.

JEL Clasification: C25, J24, J42.

\section{INTRODUCCIÓN}

El Archipiélago de Nuestra Señora del Rosario, comúnmente conocido como Las Islas del Rosario, está situado a 46 kilómetros al suroeste de Cartagena y lo conforman 23 islas de las cuales la de mayor extensión es la Isla Grande.

Las Islas del Rosario son uno de los principales atractivos turísticos de la ciudad de Cartagena, a pesar de ello, los lujosos hoteles y casas recreacionales contrastan con los altos índices de pobreza de la población nativa, que según Prieto y Acosta (1), se han incrementado como consecuencia de, entre otras variables, la insuficiencia de empleos productivos estables.

Esta insuficiencia de empleos productivos para los nativos se acentúa con la preferencia de los empresarios del turismo a contratar mano de obra desde Cartagena y la Isla de Barú (2), lo que lleva a indagar si la poca demanda laboral de nativos se explica por el bajo nivel de cualificación o si hay otras consideraciones como la raza, el género, estado civil o jefatura en el hogar.

En caso de que la educación o cualificación sean las que determinen la vinculación a un empleo productivo y estable en las Islas, entonces se consideraría al mercado de trabajo como un único mercado, en el cual es posible acceder a mejor empleo mediante el mejoramiento del capital 
humano. Pero si las consideraciones de raza, género, estado civil o jefatura en el hogar son las que determinan la vinculación a estos empleos, entonces se consideraría al mercado laboral de las Islas como un mercado segmentado.

Por un lado, en el segmento primario, moderno o protegido, se encuentran los empleos más estables, con mejor remuneración y con los requerimientos mínimos de protección social (salud y pensión); mientras que en el segundo segmento, el secundario, tradicional o de libre entrada, se encuentran aquellos empleos con más baja remuneración, sin protección social e inestabilidad y es un escape para aquellos que por razones distintas al capital humano no tienen acceso al segmento primario.

El presente artículo busca mostrar los resultados de investigación generados a partir de la caracterización del mercado laboral de las Islas del Rosario y la identificación de los factores que hacen que una persona tenga más probabilidades de obtener un empleo de calidad, es decir, con un salario superior al mínimo legal vigente y con vinculación al sistema de seguridad social. Esto con el objetivo de facilitar herramientas de apoyo para la formulación de políticas públicas que propendan la vinculación de la población nativa a empleos de mejor calidad y por esta vía lograr un mayor desarrollo para la zona. Los factores considerados en este estudio son el capital humano (educación, capacitación y experiencia) y características individuales como género, raza, estado civil y parentesco con el jefe.

El documento consta de siete secciones incluyendo la presente introducción. En la segunda, se encuentra una revisión de las teorías, seguida de una caracterización de la población de las Islas del Rosario. Finalmente se encuentra la metodología, los resultados de las estimaciones econométricas, la discusión y las conclusiones.

\section{ECONOMÍA DE LOS PEQUEÑOS TERRITORIOS INSULARES}

Los territorios insulares comparten ciertos atributos geográficos y socioeconómicos que lo distinguen de los territorios continentales. Estas características, que en su mayoría constituyen desventajas, fueron reconocidas en el documento seminal de la Conferencia de las Naciones Unidas para el Comercio y Desarrollo (UNCTAD) en 1988 (3). 
Una primera característica de los pequeños territorios insulares es su superficie reducida, lo que provoca, al igual que en cualquier pequeña economía, una limitada disponibilidad de recursos naturales y dificultades para explotar las economías de escala (3-4).

La limitada disponibilidad de recursos naturales genera una alta dependencia de las importaciones ya que los ciudadanos de estas regiones al no poder abastecerse internamente deben recurrir a la compra externa (5). Además, la poca oferta de recursos ocasiona la especialización en uno de los sectores económicos, principalmente el primario o el terciario, y dentro de este último el turismo. Por lo general, las manufacturas no sobresalen como actividad económica debido a la pérdida de competitividad que implica importar las materias primas (6-7).

Las dificultades para explotar las economías de escala desincentivan la instalación de industrias (8) y la provisión de servicios públicos (5) debido a que los costos por unidad de producción están sensiblemente por encima de los costos en los territorios continentales. No obstante, es posible aprovechar las economías de escala mediante la exportación de los excedentes, aunque la poca diversidad en la producción y la tendencia a importar, convierten a los pequeños territorios insulares en regiones muy abiertas y dependientes del mercado externo (5).

Una segunda característica de los pequeños territorios insulares es, en la mayoría de los casos, su lejanía a los grandes mercados, provocando altos costos de transportes para las personas y las mercancías (7); y mayor probabilidad de desabastecimientos y retrasos, no sólo de productos de consumo sino también de las materias primas, lo que se convierte en un punto en contra para la instalación de empresas competitivas (5).

Los territorios insulares son más propensos a sufrir de desastres naturales como huracanes, tifones o erupciones volcánicas y su poca extensión territorial hace que los daños generados por cualquier actividad económica sean superiores que en los territorios continentales. Además, estos ecosistemas son más frágiles y tienden a ser importantes fuentes de biodiversidad (3). El mercado laboral de los pequeños territorios insulares se caracteriza por poseer una escasa oferta de personal cualificado, lo que genera competencia de las empresas por atraer a los trabajadores más preparados (5). Para el caso de las Islas del Rosario, los empresarios amparados en la poca cualificación de los trabajadores nativos buscan 
mano de obra en la ciudad de Cartagena y la isla de Barú. Pero, cabria preguntar: ¿son los niveles de cualificación los que explican la inserción a los "mejores" empleos en la zona, o hay otras razones diferentes al capital humano? Al respecto, las teorías del capital humano y de la segmentación del mercado pueden dar algunas respuestas.

\section{TEORÍA DEL CAPITAL HUMANO Y SEGMENTACIÓN DEL MERCADO}

La teoría del capital humano y la teoría de la segmentación han sido ampliamente usadas para explicar la determinación de los salarios y funcionamiento del mercado de trabajo. La primera, desarrollada por Gary Becker, considera que la inversión en capital humano, especialmente en educación, mejora las destrezas y aumenta la productividad del trabajo. Esta mayor productividad se traduce en mayores salarios (9), ya que siguiendo los principios neoclásicos, la retribución a cada trabajador o tipo de trabajo es equivalente a su aportación a la producción. En el equilibrio, esta productividad es igual al nivel de salarios que se determina mediante la interacción de la oferta y demanda de trabajo (10).

Las implicaciones de esta teoría es que es posible considerar al mercado de trabajo como un mercado homogéneo, en el que la movilidad desde un empleo con precarias condiciones laborales a otro con mejores condiciones, es posible mediante el mejoramiento de las capacidades y destrezas a través de la educación y capacitación. Sin embargo, remuneraciones diferentes para un mismo tipo de trabajo hicieron que algunos economistas refutaran la teoría del capital humano (11). Dentro de estos encontramos Piore (12) y Doeringer (13); y Gordon, Edwards y Reich (14).

Para estos autores, la libre movilidad entre los sectores no es posible, ya que existen barreras a la vinculación a los "mejores" empleos, que no se explican precisamente por el nivel de educación, sino por variables institucionales, sociales e individuales como el género, la edad, la raza, la posición en el hogar y la pertenencia a un sindicato. Concluyendo entonces, que el mercado de trabajo se encuentra segmentado: un mercado primario y un mercado secundario o de libre entrada.

En el primer segmento, se encuentran los "mejores" empleos, con salarios más altos, estabilidad y protección social. Este segmento funciona con tecnología moderna y cantidades relativamente altas de capital, lo que ocasiona mayor productividad. No todos los que quieren y están 
calificados para obtener un empleo en este segmento pueden hacerlo ya que a este accede por consideraciones diferentes al capital humano (13), además, según Gordon, Edwards y Reich (14), este segmento puede estar conformado por trabajadores subordinados y trabajadores independientes.

Por su parte, en el segundo segmento, se encuentran los trabajos con salarios bajos, alta inestabilidad y rotación y la tecnología de producción es intensiva en trabajo. En este segmento no hay retornos a la educación y a la experiencia (14) y está compuesto principalmente por minorías, mujeres y jóvenes (15). Estos dos segmentos se encuentran interrelacionados: el secundario, al ser de libre entrada, es un es una alternativa para aquellos que no pueden vincularse laboralmente en el segmento primario. Esto puede verse mediante el diagrama $\mathrm{N}^{0}$ 1. En dicho diagrama, el eje horizontal representa la oferta total de trabajo. El empleo del sector primario se mide de izquierda a derecha iniciando en el punto $O_{P}$ y el empleo del sector secundario se mide de derecha a izquierda partiendo desde el punto $O_{S}$. El eje vertical de la izquierda mide el salario del sector primario (que incluye las prestaciones sociales) y el de la derecha mide el salario en el secundario. Las curvas $D_{P}$ y $D_{S}$ miden la demanda de trabajo del sector primario y el secundario, respectivamente.

Si el salario del sector primario es $W_{P 2}$, el número de trabajadores que conseguirían trabajo en este sector seria la distancia $O_{P}-N 2$, quedando $O_{S}-N 2$ disponibles para el sector secundario. Como este sector es de libre entrada, los salarios deben ser iguales a $W_{S 2}$. Supongamos ahora, un aumento en los salarios en el mercado primario a $W_{P 1}$. Esto ocasiona una disminución del empleo en este sector hasta $O_{P}-N 1$, y un aumento en la oferta en el sector secundario, que a su vez, produce una caída en los salarios en este último sector hasta $W_{S 1}$.

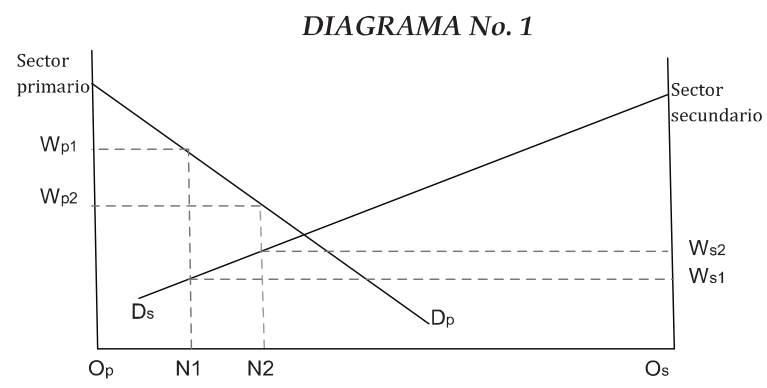

Fuente: tomado del documento "Algunos aspectos del funcionamiento del mercado laboral del sector rural" del DNP 


\section{CARACTERIZACIÓN DE LA OFERTA LABORAL DE LAS ISLAS DEL ROSARIO}

De acuerdo con el Censo Surtigas $2007^{*}$, la población de las Islas del Rosario a dicho año ascendió a 718 habitantes, de los cuales el $74,1 \%$ se concentró en Isla Grande, el 12,8\% en Isleta y el 13,1\% se distribuyó en las 21 islas restantes.

TABLA No. 1. ISLAS DEL ROSARIO. DISTRIBUCIÓN DE LA POBLACIÓN, 2007

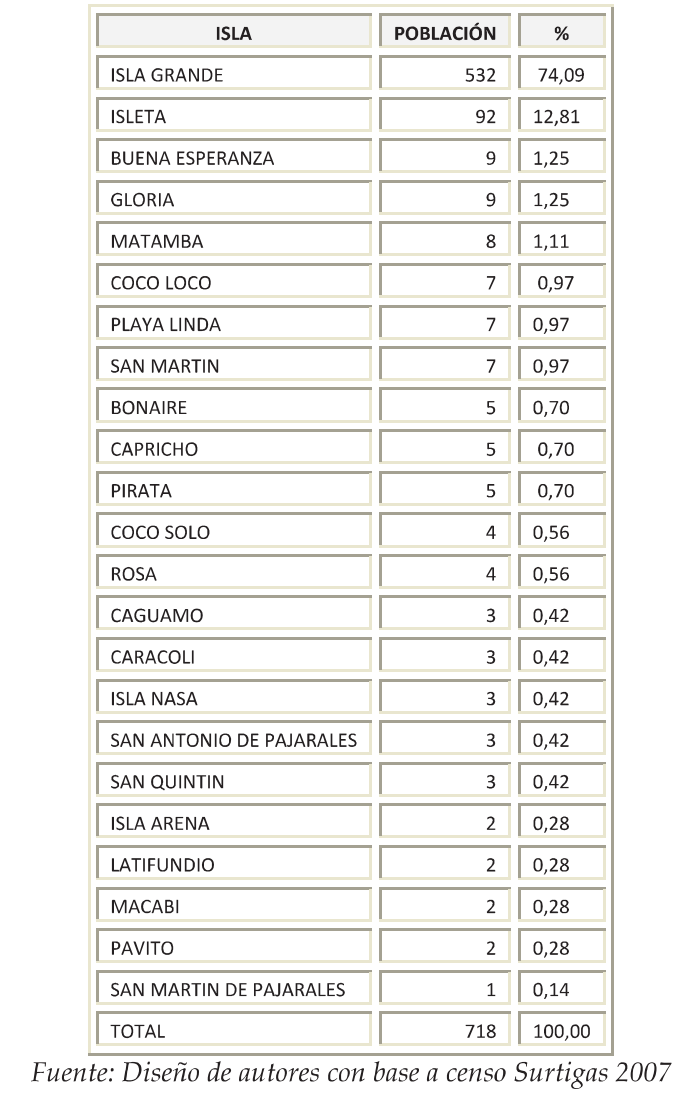

En las Islas del Rosario predomina la población masculina, que constituye el 52,5\%, mientras que la femenina constituye el 47,5\%. La edad promedio de los habitantes es de 23,5 años, siendo los de mayor concentración los menores de 10 años (26,7\% del total) y la población entre 10 y 19 años $(22,0 \%$ del total), lo que sugiere que en las Islas prevalece la población joven. El 38,4\% de la población no tiene ninguna educación y sólo el 1,1\%

* Llevado a cabo por la Fundación SURTIGAS en convenio con la Universidad de Cartagena. 
tiene una educación tecnológica o universitaria incompleta y el 0,1\% tiene educación universitaria completa.

GRÁFICA No. 1. ISLAS DEL ROSARIO. PIRÁMIDE DE POBLACIÓN, 2007

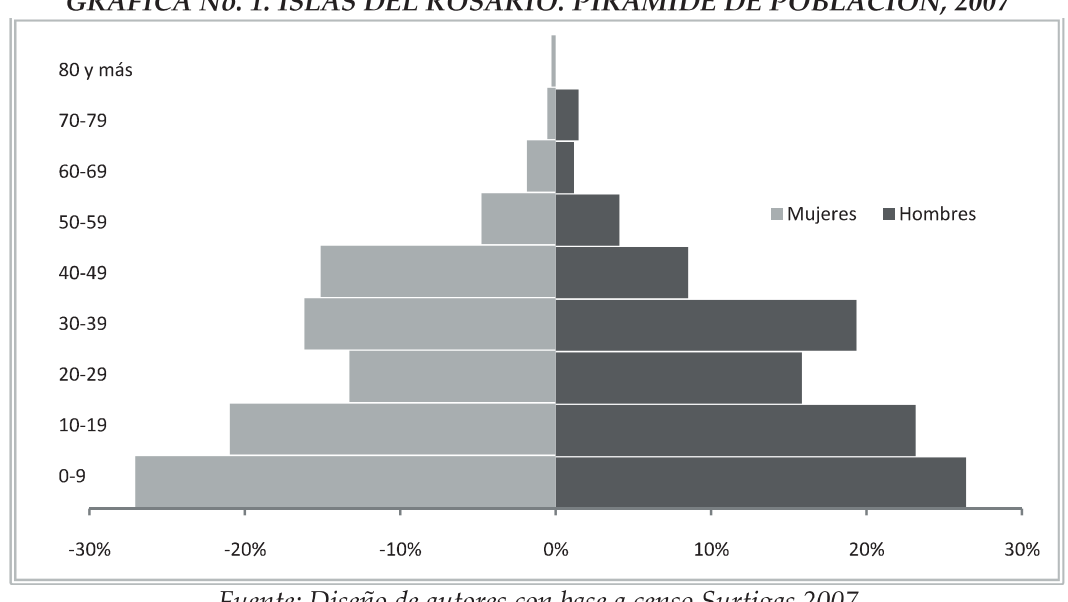

Fuente: Diseño de autores con base a censo Surtigas 2007

El grupo étnico mayoritario son los negros afrodescendientes quienes ascendieron a 320 personas y representaron el 44,6\% del total, seguidos de los raizales del archipiélago quienes totalizaron 239 personas y constituyeron el 33,3\%.

Población en edad de trabajar: La población en edad de trabajar (PET) de las Islas del Rosario, constituida por las personas mayores de 10 años*, fue de 526 personas, el 73,3\% de la población total. De estas 526 personas, el 52,2\% son hombres quienes tienen una mayor participación en las cohortes de edades comprendidas entre los 40 y 69 años. Por su parte, las mujeres representan el $47,7 \%$ de la PET y tienen mayor participación en las cohortes de edades más bajas (menores de 40 años) y en la población entre 70 y 79 años.

\footnotetext{
${ }^{*}$ Según metodología DANE, en las zonas urbanas la PET está constituida por las personas mayores de 12 años, mientras que en las zonas rurales por las personas mayores de 10 años.
} 
GRÁFICA No. 2. ISLAS DEL ROSARIO. POBLACIÓN EN EDAD DE TRABAJAR POR EDAD Y GÉNERO, 2007

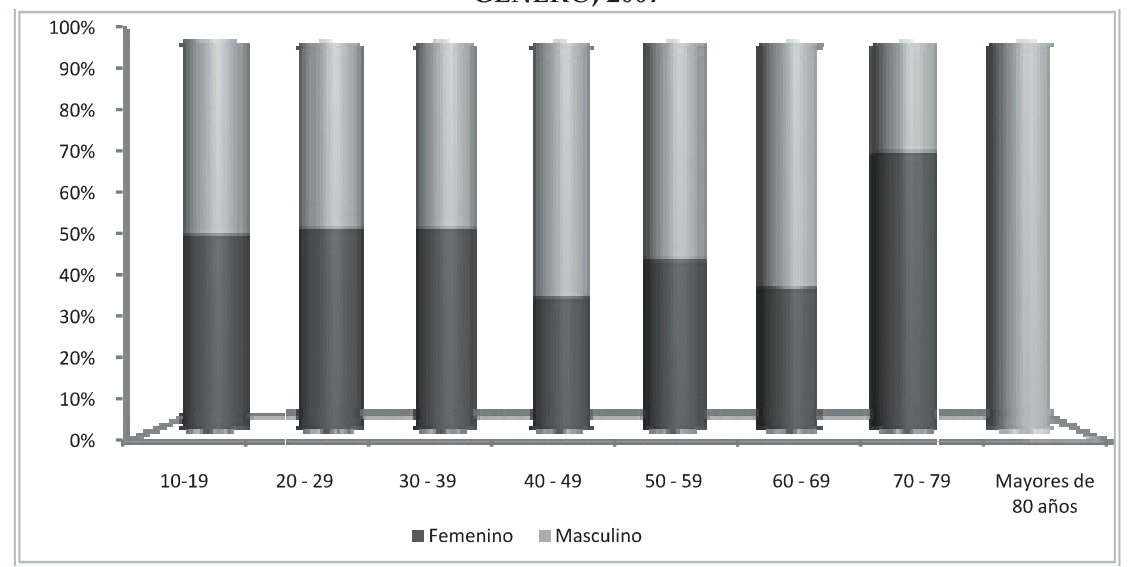

Fuente: Diseño de autores con base a censo SURTIGAS 2007

Población económicamente activa. Durante el 2007, la población económicamente activa (PEA) de las Islas del Rosario, constituida por aquellas personas en edad de trabajar que trabajan o están buscando empleo, fue de 254 personas, el 48,1\% de la población en edad de trabajar (Tasa global de participación, TGP) y el 35,4\% de la población total (Tasa bruta de participación, TBP).

Por género, se destaca que el $68,9 \%$ de la PEA son hombres, quienes son más del $50 \%$ en todas las cohortes de edad. A su vez, para este grupo tanto la TBP $(46,4 \%)$ como la TGP $(63,6 \%)$ duplican las tasas de las mujeres, las cuales fueron del $23,2 \%$ y $31,5 \%$ respectivamente.

Por rango de edad, el 61,1\% de la PEA se encuentra entre los 30 y 49 años. Específicamente, el 33,5\% de la PEA tiene entre 30 y 39 años y el 27,6\% tiene entre 40 y 49 años.

En la gráfica No. 3, se muestra la TGP por rangos de edad y género. De este se deduce que en términos generales la TGP aumenta con la edad y que en todas las cohortes de edad la TGP de los hombres son superiores a las tasas de las mujeres. A su vez, las mujeres entre 50 y 59 años presentaron la mayor TGP, la cual fue de 57,1\%, mientras que para los hombres la mayor tasa de participación se presenta para edades superiores a 60 años. 
GRÁFICA No. 3. ISLAS DEL ROSARIO. TASA GLOBAL DE PARTICIPACIÓN POR RANGOS DE EDAD Y GÉNERO, 2007

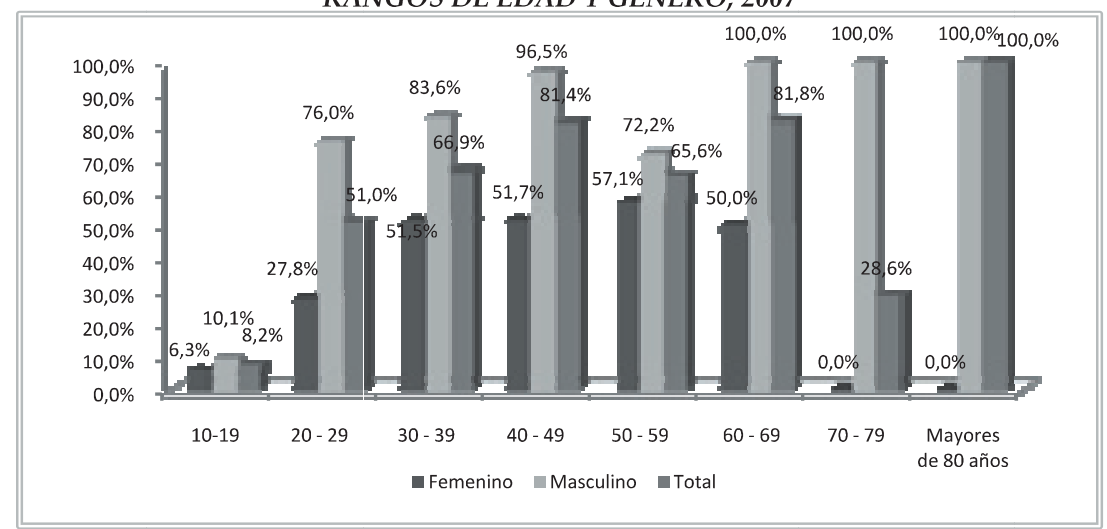

Fuente: Diseño de autores con base a censo Surtigas 2007

De la gráfica No. 4 se observa que la TGP aumenta a medida que lo hace el nivel educativo, principalmente a partir de la educación primaria completa. Tanto para los hombres como las mujeres, la TGP es mayor para aquellos quienes tienen una educación tecnológica o universitaria incompleta.

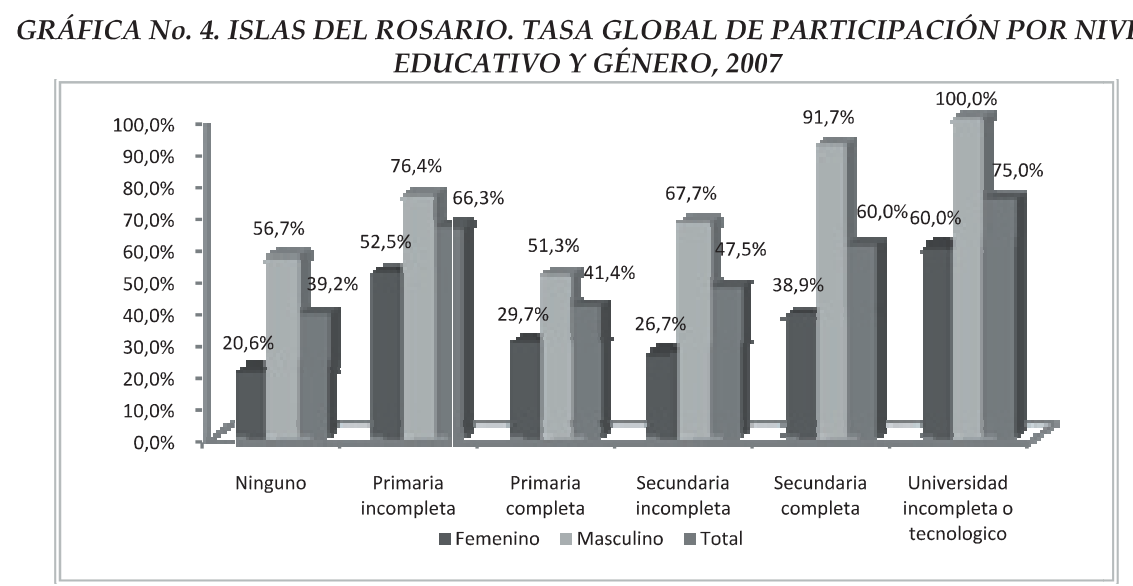

Fuente: Diseño de autores con base a censo SURTIGAS 2007

Población desocupada: Durante el Censo de Surtigas 2007, se encontraron 17 personas desempleadas, de las cuales 9 son hombres y 8 mujeres. Lo anterior se expresa en una tasa de desempleo (TD) del 6,7\%, y una tasa del $10,1 \%$ para las mujeres y de $5,1 \%$ para los hombres. En las mujeres las mayores TD se presenta en aquellas que no tienen ningún nivel educativo, mientras que en los hombres se presentan en aquellos que tienen una educación secundaria incompleta. A su vez, la población más joven (entre 
10 y 19 años) es la más afectada con el desempleo, situación que tiende a mejorar con la edad.

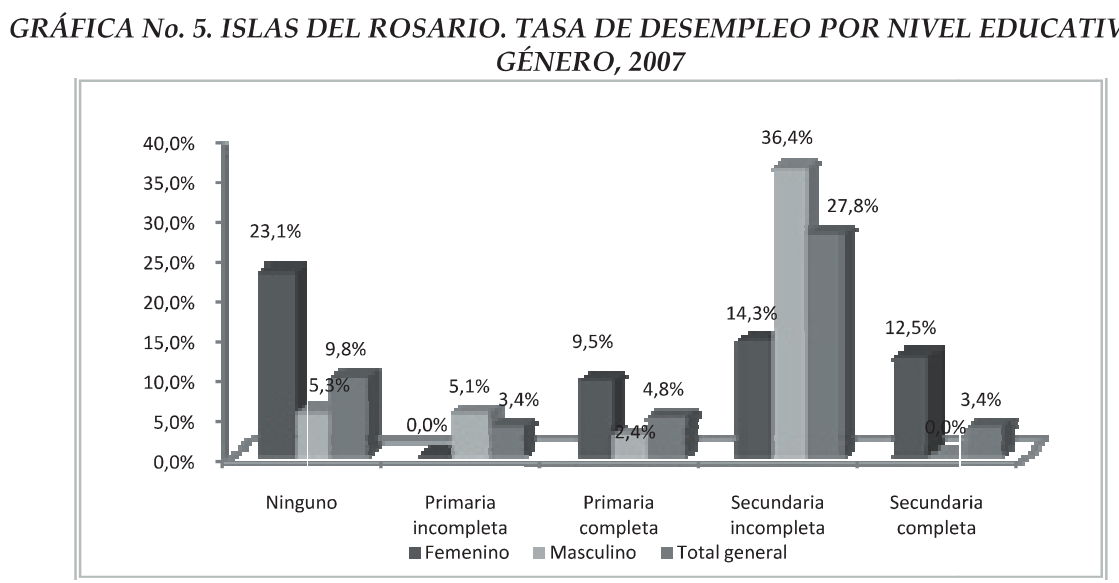

Fuente: Diseño de autores con base a censo Surtigas 2007

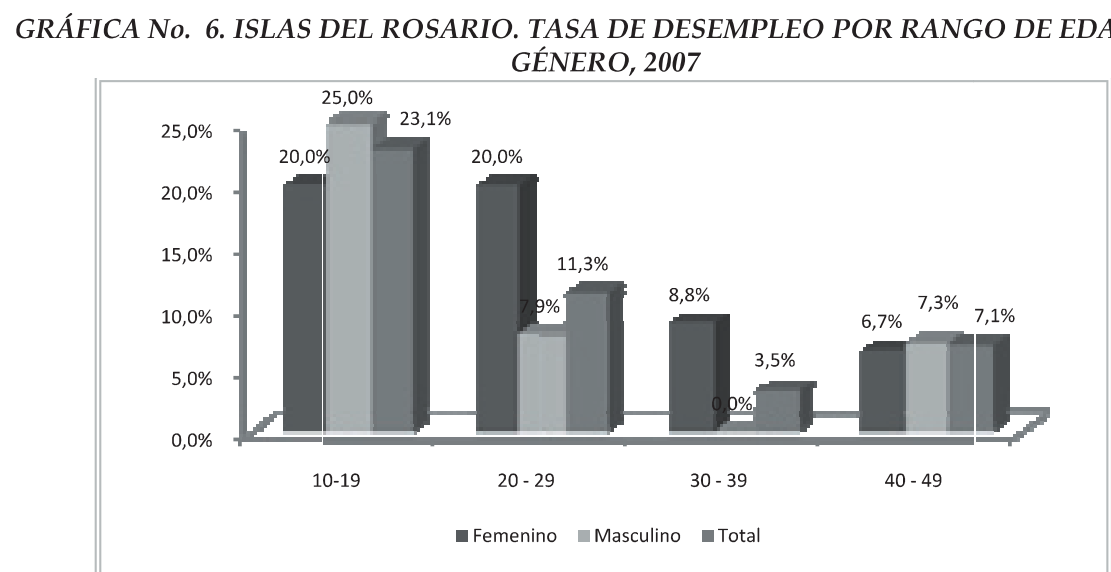

Fuente: Diseño de autores con base a censo Surtigas 2007

Según posición en el hogar, los cabezas de hogar tienen una TD más alta, a saber, del $4,2 \%$, seguido de los conyugues con una tasa de $2,9 \%$ y la de los hijos la cual es del $2,4 \%$.

El $52,9 \%$ de los desempleados fueron personas autoreconocidas como raizales del archipiélago, quienes mostraron una TD del 9,9\%, mientras que los negros afrodescendientes constituyen el 41,2\% de los desempleados con una TD de 6,9\%. Quienes se autoreconocieron como mestizos o de otras etnias, mostraron una TD del 1,6\%. 
Población ocupada: De acuerdo con el censo Surtigas 2007, la población ocupada de las Islas del Rosario fue de 237 personas, lo que constituye el $45,1 \%$ de la PET (tasa de ocupación, TO). Por género, se encuentra que de las 237 personas ocupadas, el 70,0\% son hombres, quienes tienen una TO del 60,4\%; mientras que las mujeres constituyen el 30,0\% de los ocupados, con una TO del $28,3 \%$. Por rango de edad, se observa que la población entre 30 y 39 años conforma el 34,6\% de los ocupados, seguido de la población entre 40 y 49 años quienes participan con el $27,4 \%$.

$\mathrm{Al}$ considerar la TO por rangos de edad y género, se observa que muestra un comportamiento similar a la TGP, aumentando a medida que lo hace la edad y siendo mayor en los hombres en todos los rangos de edad. Las mujeres muestran las TO más altas en el rango de edad de 50 a 59 años, la cual fue de $57,1 \%$, mientras que en los hombres la mayor TO se muestran para los mayores de 60 años.

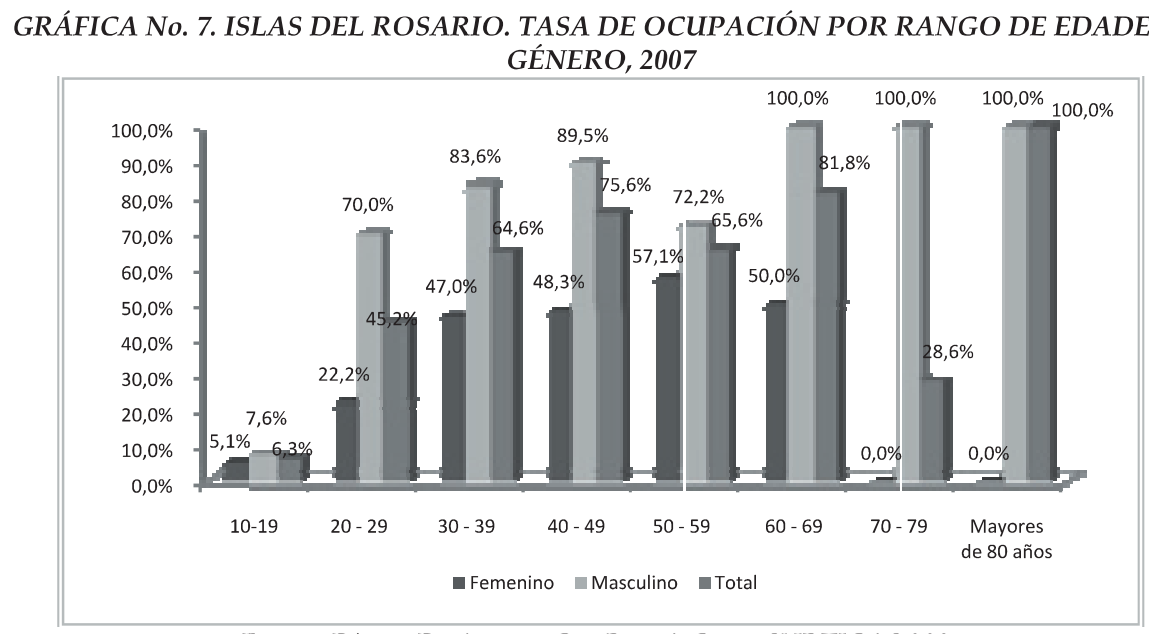

Fuente: Diseño De Autores Con Base A Censo SURTIGAS 2007

Por nivel educativo se destaca que los ocupados tienen un bajo nivel de cualificación, ya que el $90,7 \%$ de ellos no poseen siquiera una educación secundaria completa. Además, tanto los hombres como las mujeres, la mayor TO se presenta en personas con una educación tecnológica o universitaria incompleta. 


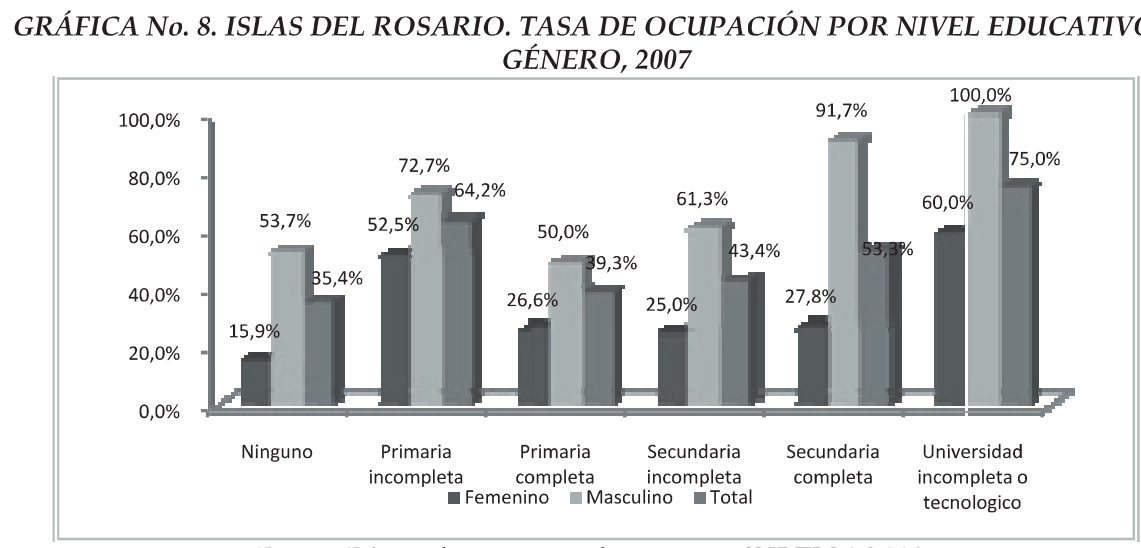

Fuente: Diseño de autores con base a censo SURTIGAS 2007

Según posición en el hogar, la TO más alta es la del jefe de hogar $(86,8 \%)$, seguida de la de otros parientes $(46,2 \%)$ y la de los conyugues $(40,7 \%)$.

Al descomponer los ocupados según actividad económica, se encuentra que el sector que más empleo genera es el de servicios $(39,7 \%)$, seguido del turismo $(19,4 \%)$ y el comercio $(8,0 \%)$. Se destaca que en la zona no hay ningún ocupado en el sector industria.

TABLA No. 2. ISLAS DEL ROSARIO. OCUPADOS POR ACTIVIDAD ECONÓMICA

\begin{tabular}{|l|r|r|r|r|r|r|r|}
\hline \multicolumn{1}{|c|}{ Sector } & \multicolumn{1}{c}{ Femenino } & \multicolumn{1}{c|}{ Masculino } & \multicolumn{2}{c|}{ Total } \\
\hline Agrícola & 0 & $0,0 \%$ & 1 & $0,6 \%$ & 1 & $0,4 \%$ \\
\hline Pecuaria & 1 & $1,4 \%$ & 14 & $8,4 \%$ & 15 & $6,3 \%$ \\
\hline Comercio & 12 & $16,9 \%$ & 7 & $4,2 \%$ & 19 & $8,0 \%$ \\
\hline Servicios & 25 & $35,2 \%$ & 69 & $41,6 \%$ & 94 & $39,7 \%$ \\
\hline Turismo & 13 & $18,3 \%$ & 33 & $19,9 \%$ & 46 & $19,4 \%$ \\
\hline Otra actividad & 16 & $22,5 \%$ & 38 & $22,9 \%$ & 54 & $22,8 \%$ \\
\hline No informa & 4 & $5,6 \%$ & 4 & $2,4 \%$ & 8 & $3,4 \%$ \\
\hline Total general & $\mathbf{7 1}$ & $\mathbf{1 0 0 , 0} \%$ & $\mathbf{1 6 6}$ & $\mathbf{1 0 0 , 0} \%$ & $\mathbf{2 3 7}$ & $\mathbf{1 0 0 , 0} \%$ \\
\hline
\end{tabular}

Al sector servicios se dedican principalmente hombres entre 30 y 49 años de edad (46,8\% de los ocupados del sector), quienes a su vez, constituyen el $24,1 \%$ del total de ocupados en las Islas del Rosario, aproximadamente la cuarta parte. Por otro lado, al turismo se dedican hombres en este mismo rango de edad, quienes constituyen el 47,5\% del sector y el 8,8\% del total de ocupados; y al sector comercio se dedican mujeres entre 20 y 39 años, quienes representan el $42,9 \%$ de los ocupados del sector y el 3,8\% del total de ocupados. 
De acuerdo a la posición ocupacional, se encuentra que los asalariados en las Islas son el 63,7\% del total de ocupados (35,0\% cuidanderos, 20,3\% empleados y obreros particulares, 3,8\% de empleados públicos, 3,0\% de jornaleros o peón y $1,7 \%$ de empleados domésticos). Por su parte, los trabajadores independientes representan el $29,1 \%$ (28,3\% trabajadores por cuenta propia y el $0,8 \%$ patrones o empleadores). Los trabajadores familiares sin remuneración representan el 4,6\%.

En la tabla No. 3, se muestran algunas variables que dan idea de la calidad de empleos. De ella se concluye que las condiciones de trabajo de la población de las Islas del Rosario son precarias, ya que sólo el 56,3\% de los asalariados tiene un contrato de trabajo, situación que se agrava si se considera que sólo el 33,8\% tiene los requerimientos mínimos de protección social (salud y pensión). Al considerar a los trabajadores independientes, se encuentra que el 78,3\% de ellos gana menos de $1 \mathrm{SMLV}$, lo que incide en que sólo el 10,1\% de ellos tengan acceso a salud y pensiones.

TABLA No. 3. ISLAS DEL ROSARIO. CALIDAD DE EMPLEO, 2007

\begin{tabular}{|c|c|c|c|c|c|c|c|c|c|c|c|c|}
\hline \multirow[b]{2}{*}{ Variable } & \multicolumn{6}{|c|}{ Asalariados } & \multicolumn{6}{|c|}{ Independientes } \\
\hline & \multicolumn{2}{|c|}{ Femenino } & \multicolumn{2}{|c|}{ Masculino } & \multicolumn{2}{|c|}{ Total } & \multicolumn{2}{|c|}{ Femenino } & \multicolumn{2}{|c|}{ Masculino } & \multicolumn{2}{|r|}{ Total } \\
\hline \multicolumn{13}{|l|}{ Contrato laboral } \\
\hline Sin contrato & 18 & $46,2 \%$ & 48 & $42,9 \%$ & 66 & $43,7 \%$ & - & - & - & - & - & - \\
\hline Término fijo & 11 & $28,2 \%$ & 35 & $31,3 \%$ & 46 & $30,5 \%$ & - & - & - & - & - & - \\
\hline Indefinido & 10 & $25,6 \%$ & 29 & $25,9 \%$ & 39 & $25,8 \%$ & - & - & - & - & - & - \\
\hline Total & 39 & $100,0 \%$ & 112 & $100,0 \%$ & 151 & $100,0 \%$ & - & - & - & - & - & - \\
\hline \multicolumn{13}{|l|}{ Seguridad social } \\
\hline Ni salud, ni pensión & 7 & $17,9 \%$ & 20 & $17,9 \%$ & 27 & $17,9 \%$ & 6 & $27,3 \%$ & 10 & $21,3 \%$ & 16 & $23,2 \%$ \\
\hline Salud o pensión & 22 & $56,4 \%$ & 51 & $45,5 \%$ & 73 & $48,3 \%$ & 13 & $59,1 \%$ & 33 & $70,2 \%$ & 46 & $66,7 \%$ \\
\hline Salud y pensión & 10 & $25,6 \%$ & 41 & $36,6 \%$ & 51 & $33,8 \%$ & 3 & $13,6 \%$ & 4 & $8,5 \%$ & 7 & $10,1 \%$ \\
\hline Total & 39 & $100,0 \%$ & 112 & $100,0 \%$ & 151 & $100,0 \%$ & 22 & $100,0 \%$ & 47 & $100,0 \%$ & 69 & $100,0 \%$ \\
\hline \multicolumn{13}{|l|}{ Ingreso laboral } \\
\hline Menos de 1 SMLV & 22 & $56,4 \%$ & 51 & $45,5 \%$ & 73 & $48,3 \%$ & 19 & $86,4 \%$ & 35 & $74,5 \%$ & 54 & $78,3 \%$ \\
\hline Entre 1 y 2 SMLV & 16 & $41,0 \%$ & 55 & $49,1 \%$ & 71 & $47,0 \%$ & 2 & $9,1 \%$ & 8 & $17,0 \%$ & 10 & $14,5 \%$ \\
\hline Más de 2 SMLV & 1 & $2,6 \%$ & 6 & $5,4 \%$ & 7 & $4,6 \%$ & 1 & $4,5 \%$ & 4 & $8,5 \%$ & 5 & $7,2 \%$ \\
\hline Total & 39 & $100,0 \%$ & 112 & $100,0 \%$ & 151 & $100,0 \%$ & 22 & $100,0 \%$ & 47 & $100,0 \%$ & 69 & $100,0 \%$ \\
\hline
\end{tabular}

Al considerar el subempleo subjetivo, se encuentra que el 50,6\% de los ocupados no está satisfecho con su remuneración, mientras que el 37,1\% considera que no se siente satisfecho con su trabajo porque no desarrolla sus capacidades. 
Población inactiva: La población inactiva de las Islas de Rosario ascendió a 272 personas, quienes representan el 51,7\% de la PET. La principal causa de inactividad fue el estudio (44,1\%), seguida de las labores en el hogar $(31,6 \%)$, lo que va acorde con el parentesco con respecto al jefe, ya que el $57,0 \%$ de la PEI son hijos y $29,0 \%$ conyugues. Por género, se destaca que las mujeres constituyen el $63,2 \%$ de la PEI, quienes a su vez, representan el $68,5 \%$ de las mujeres en edad de trabajar. Ellas se dedican principalmente a las labores del hogar (46,5\%), seguida de las que estudian (35,5\%). Por su parte, los hombres constituyen el $36,8 \%$ de la PEI, quienes representan el $36,4 \%$ de los hombres en edad de trabajar y su principal causa de inactividad es el estudio.

TABLA No. 4. ISLAS DEL ROSARIO. RAZÓN DE INACTIVIDAD POR GÉNERO, 2007
\begin{tabular}{|l||r|r|r|r|r|r|r||}
\hline \multicolumn{1}{|c|}{ Razón de Inactividad } & Femenino & Masculino & Total \\
\hline Estudiando & 61 & $35,5 \%$ & 59 & $59,0 \%$ & 120 & $44,1 \%$ \\
\hline Realizando oficios del hogar & 80 & $46,5 \%$ & 6 & $6,0 \%$ & 86 & $31,6 \%$ \\
\hline Incapacitado permanente para trabajar sin pensión & 0 & $0,0 \%$ & 1 & $1,0 \%$ & 1 & $0,4 \%$ \\
\hline Realizando actividades de autoconsumo & 1 & $0,6 \%$ & 1 & $1,0 \%$ & 2 & $0,7 \%$ \\
\hline Otra Actividad & 30 & $17,4 \%$ & 33 & $33,0 \%$ & 63 & $23,2 \%$ \\
\hline Total Fuente: Diseño de autores con base a censo SURTIGAS 2007 & 172 & $100,0 \%$ & 100 & $100,0 \%$ & 272 & $100,0 \%$ \\
\hline
\end{tabular}

Por rango de edad, se encuentra que la población entre 10 y 19 años representa el 53,3\% de la PEI, seguida de la población entre 20 y 29 años, quienes constituyen el 18,8\%.

\section{METODOLOGÍA}

Con base en la información obtenida del Censo Surtigas 2007 realizado a la población perteneciente a las 23 islas que conforman el Archipiélago del Rosario, se construyó un modelo de elección discreta tipo probit que explica de qué factores depende la probabilidad de que un ocupado tenga un empleo en el sector primario:

$$
Y_{i}=P_{i}=P\left(Y_{i}=1\right)=F\left(X_{i} \beta\right)=\varphi\left(X_{i} \beta\right)
$$

Que sigue una función normal estándar acumulativa:

$$
F=\frac{1}{\sqrt{2 \pi}} \int_{-\infty}^{X_{i} \beta} e^{-Z^{2} / 2} d z
$$

Donde $Z \sim N\left(0, \sigma^{2}\right)$ 
La variable dependiente es dicótoma, asumiendo el valor de 1 para aquellos que tienen un empleo en el segmento primario y 0 para aquellos que tienen un empleo en el segmento secundario. En este trabajo se considera a los trabajadores del primer segmento a aquellos que ganan un salario superior o igual al mínimo legal vigente y que además cuentan con los requisitos mínimos de seguridad social (salud y pensiones) y se considera como ocupado en el segmento secundario a aquellos que no cuentan con condiciones laborales exigidas por la normatividad en términos de salario mínimo, salud y pensiones.

Las variables independientes se encuentran a continuación en la tabla No. 5 .

TABLA No. 5. VARIABLES DEL MODELO ECONOMÉTRICO

\begin{tabular}{|c|c|c|}
\hline & VARIABLE & INDICADOR \\
\hline 1 & EDUCACIÓN & Años de educación \\
\hline 2 & EDAD & Años de edad \\
\hline 3 & EDAD AL CUADRADO & Cuadrado de la edad \\
\hline 4 & CAPACITACIÓN & $\begin{array}{l}1 \text { si ha recibido capacitación } \\
0 \text { si no la ha recibido }\end{array}$ \\
\hline 5 & RESIDENCIA & Años de residencia en las Islas \\
\hline 6 & RAIZAL & $\begin{array}{l}1 \text { si se autoreconoce como Raizal } \\
0 \text { en otro caso }\end{array}$ \\
\hline 7 & GENERO & $\begin{array}{l}1 \text { si es hombre } \\
0 \text { si es mujer }\end{array}$ \\
\hline 8 & CON CONYUGE & $\begin{array}{l}1 \text { si es casado o convive en unión libre } \\
0 \text { en otro caso }\end{array}$ \\
\hline 9 & JEFATURA DEL HOGAR & $\begin{array}{l}1 \text { si es jefe de hogar } \\
0 \text { en otro caso }\end{array}$ \\
\hline 10 & TURISMO & $\begin{array}{l}1 \text { Si está empleado en el sector turismo } \\
0 \text { en otro caso }\end{array}$ \\
\hline
\end{tabular}

Para corregir el posible sesgo de selección que se origina de correr el modelo sólo con la población ocupada, se usará el método de corrección en dos etapas propuesto por Heckman (18):

- Estimar la probabilidad de que una persona en edad de trabajar tenga un empleo en el segmento primario, mediante un modelo probit. En este modelo, la variable dependiente toma el valor de 1 si la persona tiene un empleo con seguridad social y un salario superior o igual al mínimo y 0 en caso contrario. Las variables independientes son: años de EDUCACIÓN; años de RESIDENCIA en las Islas; EDAD en años; 
EDAD AL CUADRADO; y las siguientes dummies: CAPACITACIÓN, RAIZAL, GÉNERO, JEFE y CON CONYUGE. De este modelo, se obtiene para cada observación, la razón inversa de Mills $\lambda$ i que se interpreta como la probabilidad de que el i-ésimo individuo sea ocupado y tenga un empleo de calidad.

- Se estima el modelo probit de la probabilidad de que un ocupado tenga un empleo en el segmento primario, añadiéndole la variable razón inversa de Mills, la cual corrige los posibles problemas de sesgo de selección.

Debido a que el modelo probit se estima mediante el método de máxima verosimilitud, se usará el estadístico Razón de verosimilitud para evaluar la significancia global del modelo, los estadísticos $\mathrm{z}$ para probar la significancia individual de los parámetros y el R-cuadrado de Mcfadden para examinar su bondad de ajuste. Además, como los coeficientes de este tipo de modelos no se pueden interpretar directamente, se calculan los efectos marginales de una persona promedio.

Dado que es posible encontrar problemas de heteroscedasticidad ya que la información es de corte transversal, se correrá el modelo con errores estándar y varianzas consistentes con heteroscedasticidad de White.

Finalmente, para probar la teoría del capital humano, las variables relacionadas con el capital humano tales como educación, experiencia y capacitación deben ser significativas, mientras que las características individuales del ocupado, tales como género, raza, jefatura en el hogar y estado civil deben ser no significativas. Si estas últimas son significativas, se comprobaría la hipótesis de la segmentación del mercado laboral.

\section{RESULTADOS ECONOMÉTRICOS}

En el ejercicio de modelación se corrieron 2 modelos, omitiendo en el último las variables de jefatura del hogar y unión, ya que no sólo no eran significativas, sino que también presentaban signos incorrectos y generaban problemas en otras variables.

La última de estas modelaciones se presenta en la tabla No. 6, la cual está corregida del sesgo de selección mediante el método propuesto por Heckman y con errores estándar y varianzas consistentes con 
heteroscedasticidad de White. De este modelo se infiere que todas las variables en su conjunto explican la probabilidad de pertenecer a un empleo con condiciones mínimas laborales ya que la razón de verosimilitud $(-96,0)$ es significativa al $1 \%$. Además, todas las variables resultaron significativas a un nivel de significancia del $5 \%$ y presentaron los signos esperados.

El modelo tiene buena capacidad predictiva debido a que acertó el 78,9\% de los casos, es decir, estimó una probabilidad cercana a cero cuando el valor real era cero y estimó valores cercanos a 1 cuando el valor real era 1. Por su parte, el R- cuadrado de Mcfadden es de $22,9 \%$ el cual es relativamente bajo, aunque hay que tomar en cuenta que los datos de corte transversal por lo general presentan bajos R- cuadrados. Y finalmente la probabilidad de la Razón inversa de Mill indica que la corrección por el sesgo de selección es adecuada a un nivel de significancia del 7\%.

TABLA No. 6. RESULTADOS DEL MODELO PROBIT.

\begin{tabular}{|c|c|c|c|c|c|}
\hline Variable & Coeficiente & Error Estandar & Efecto Marginal $^{*}$ & Estadístico z & Probabilidad \\
\hline Género & 1,55 & 0,51 & 0,212 & 3,01 & 0,00 \\
\hline Años de Residencia & $-0,03$ & 0,01 & $-0,006$ & $-2,91$ & 0,00 \\
\hline Raizal & $-0,92$ & 0,36 & $-0,148$ & $-2,51$ & 0,01 \\
\hline Años de Educación & 0,28 & 0,08 & 0,053 & 3,47 & 0,00 \\
\hline Edad & 0,48 & 0,14 & 0,091 & 3,59 & 0,00 \\
\hline Edad al cuadrado & $-0,01$ & 0,00 & $-0,001$ & $-3,55$ & 0,00 \\
\hline Capacitación & 1,02 & 0,41 & 0,260 & 2,47 & 0,01 \\
\hline Turismo & 0,63 & 0,24 & 0,148 & 2,66 & 0,01 \\
\hline Razón de Mill & $-5,29$ & 2,34 & - & $-2,26$ & 0,02 \\
\hline Constante & $-12,34$ & 3,07 & - & $-4,02$ & 0,00 \\
\hline$R V=-96,0$ & & & \multicolumn{3}{|c|}{ Cuenta R- Cuadrado $=78,9 \%$} \\
\hline \multicolumn{6}{|c|}{ R-cuadrado McFadden= 22,98\% } \\
\hline
\end{tabular}

Al examinar las variables relacionadas con la teoría del capital humano, se encuentra que una persona que se capacita tiene un $26,0 \%$ más de probabilidad de obtener un empleo en el segmento primario que quien no lo hace, la cual es la probabilidad más alta del modelo.

Por su parte, partiendo desde el nivel de educación promedio (4,5 años), un año adicional de educación formal aumenta la probabilidad en un 5,3\% de obtener un empleo en el segmento primario, probabilidad que se hace mayor a medida que partimos de niveles más altos de educación (gráfica 
$\mathrm{N}^{0}$ 9). Así que una persona con 11 años de educación tiene un $72,9 \%$ de probabilidades de pertenecer al segmento primario.

La edad (proxy de la experiencia), también afecta de manera positiva la probabilidad de estar vinculado a un empleo en el segmento primario, aunque contrario a la educación, el efecto de la edad se va disipando a niveles mayores como lo indica el coeficiente negativo de la edad al cuadrado y la gráfica $\mathrm{N}^{\circ} 10$.

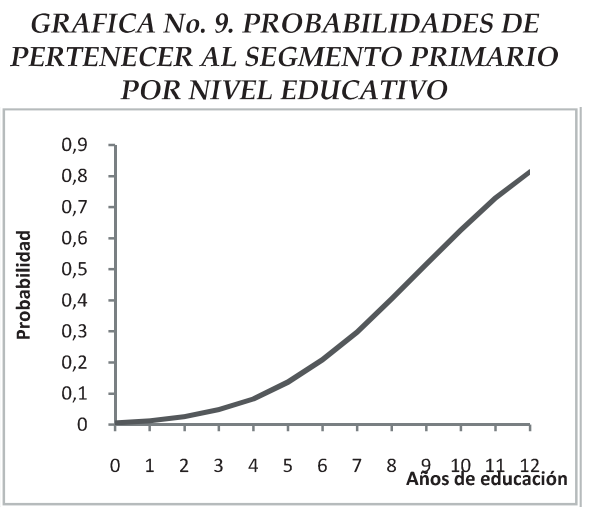

Fuente: cálculo de autoras
GRAFICA No. 10. PROBABILIDADES DE PERTENECER AL SEGMENTO PRIMARIO POR EDAD

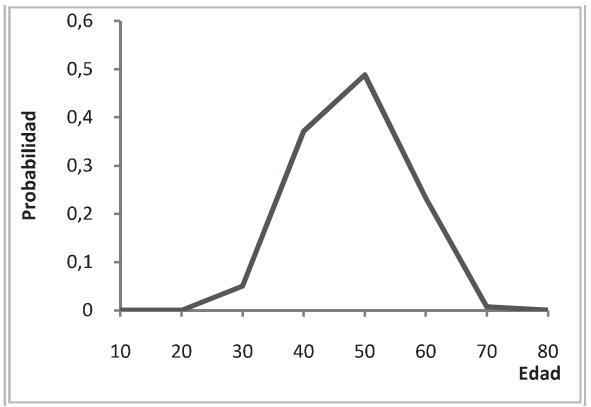

Fuente: cálculo de autoras

Al examinar las variables relacionadas con la teoría de la segmentación, se encuentra la probabilidad de obtener un empleo en el segmento primario tiene una relación inversa con los años de residencia en las Islas y con la variable raizal, demostrando así la preferencia de los empresarios por contratar mano de obra proveniente de otras regiones y comprobando la hipótesis de segmentación por raza. Esto implica que un raizal con el mismo nivel de capital humano que una persona perteneciente a otro grupo étnico, tiene menos probabilidades de obtener un empleo con un salario igual o superior al mínimo y con seguridad social. En la grafica $\mathrm{N}^{\mathrm{o}}$ 11 y 12, se observa dicha situación. 
GRAFICA No. 11. PROBABILIDADES DE PERTENECER AL SECTOR PRIMARIO POR RAZA Y NIVEL EDUCATIVO

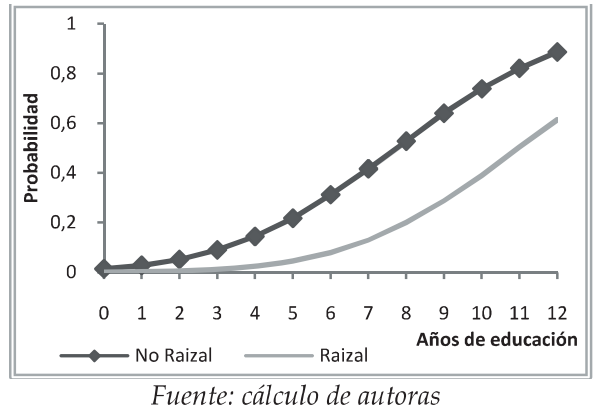

GRAFICA No. 12. PROBABILIDADES DE PERTENECER AL SECTOR PRIMARIO POR RAZA Y EDAD

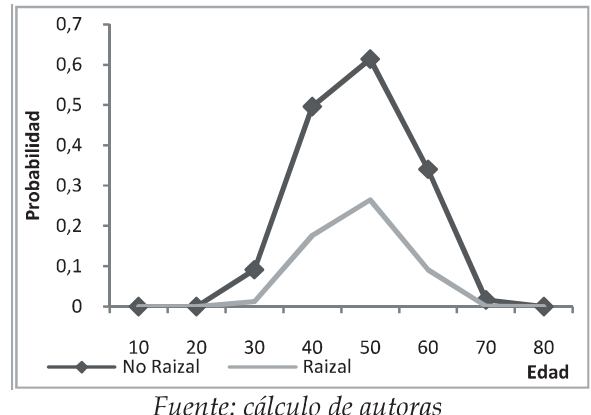

A la segmentación por raza se le suma la segmentación por género, lo que indica que una mujer con el mismo nivel de capital humano que un hombre tiene más dificultades para acceder a un empleo en el segmento primario, como se observa en las graficas No. 13 y 14.

GRAFICA N ${ }^{\circ}$ 13. PROBABILIDADES DE PERTENECER AL SECTOR PRIMARIO POR GÉNERO Y NIVEL EDUCATIVO

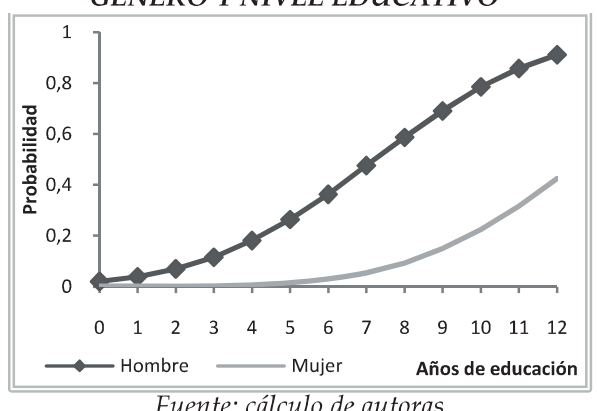

Fuente: cálculo de autoras
GRAFICA N $N^{\circ}$ 14. PROBABILIDADES DE PERTENECER AL SECTOR PRIMARIO POR GÉNERO Y EDAD

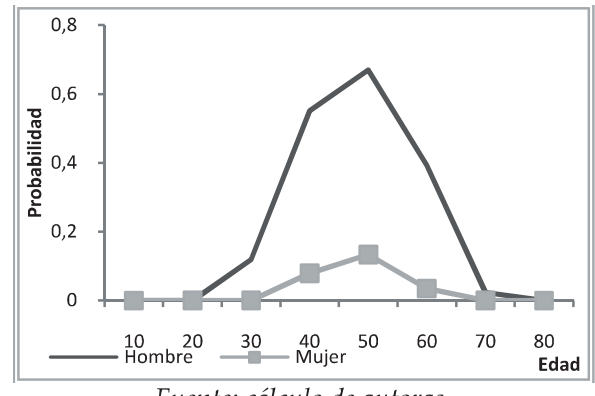

Fuente: cálculo de autoras

Se destaca que tanto en los raizales y las mujeres la brecha con respecto a los no raizales y los hombres tienden a ampliarse con el mayor nivel educativo, siendo las brechas más marcadas entre las mujeres y los hombres que entre los raizales y no raizales. Por su parte, hay que considerar que los jóvenes también tienden a ser excluidos del mercado primario de las islas, debido a la poca experiencia laboral que tienen. 


\section{DISCUSIÓN}

De los resultados de la modelación se infiere que la educación, la experiencia y la capacitación tienen retornos positivos en Islas del Rosario ya que estas aumentan la probabilidad de vinculación a un empleo con condiciones mínimas de calidad, lo que va acorde con los postulados de la teoría del capital humano.

Lo anterior sugiere que el principal obstáculo de la población nativa para acceder a mejores empleos es su poca cualificación. Sin embargo, el único criterio para acceder a un empleo de calidad no es el capital humano, ya que las mujeres, los jóvenes y los raizales tienen más dificultades de acceder a un empleo de este tipo, lo que respalda la teoría de la segmentación. Esto implica que el mercado laboral de las Islas del Rosario puede ser descompuesto en un segmento primario, en el que se encuentran los "mejores" empleos y está integrado principalmente por hombres, no raizales y personas con experiencia; y un segmento secundario, en el que prevalecen los empleos con condiciones laborales precarias y al que se dedican principalmente mujeres, jóvenes y raizales.

Por otra parte, debido a las características de los pequeños territorios insulares, en la que las actividades industriales no pueden desarrollarse, y dadas las restricciones que existen para las actividades de pesca, se destaca la importancia del turismo como el sector capaz de generar empleos productivos para los nativos.

\section{CONCLUSIONES}

El mercado laboral de las Islas del Rosario se caracteriza por presentar bajas tasas de desempleo con relación a Cartagena, pero las condiciones laborales son precarias como lo demuestran las altas tasas de subempleo subjetivo y la alta proporción de personas que están empleadas sin contar con los requisitos mínimos de la legislación laboral (salud, pensiones y salario mínimo).

Se destaca la importancia de la capacitación para el trabajo y de la educación formal como mecanismo para garantizar la creación o el acceso a empleos de mejor calidad y por esta vía generar un desarrollo social y económico incluyente para la zona objeto de estudio. Esto lleva a sugerir la 
formulación de políticas públicas que promuevan el entrenamiento del capital humano.

Por otra parte, dado que se comprueba la hipótesis de la segmentación por género, raza y edad, se concluye que deben promoverse políticas públicas encaminadas a lograr igualdad de oportunidades para las mujeres, raizales y los jóvenes al ser ellos los más afectados por la poca demanda laboral de nativos por parte de las unidades productivas en las Islas.

A su vez, dada la incapacidad del sector primario para desarrollarse y del sector industrial para establecerse en estos territorios insulares, la absorción de la mano de obra local debe generarse en el sector turismo. Sin embargo, el sector formal del turismo no podrá absorber toda la mano de obra nativa, por tanto se plantea la necesidad de establecer estrategias para la organización y capacitación de aquellos que tienen sustento indirecto del turismo en busca de mejorar sus condiciones laborales.

\section{REFERENCIAS BIBLIOGRÁFICAS}

1. Prieto W, Acosta J. Desarrollo local en las islas del Rosario. Cartagena: Universidad de Cartagena, Departamento De Investigaciones Económicas y Sociales DIES, Revista Panorama económico. 2007; (15): 43-114.

2. Duran C. Isla Grande: Colonización y descolonización del régimen de representación sobre el territorio y la población de la isla. [Online]. Bogotá: Universidad de los Andes, 2005. Disponible en: http:/ / elcentro.uniandes.edu.co/cr/antropologia/caduran/islagra nde/islagrande.doc.

3. Briguglio L. Small island developing states and their economic vulnerabilities. Word development. 1995; 23 (9).

4. Godenau D, Hernández R. Insularidad: ¿Un concepto de relevancia analítica?. Universidad de La Laguna. Estudios Regionales. 1996; 45 (45): 177-192.

5. Sánchez A. Modelo para la medición del capital intelectual de territorios insulares: una aplicación al caso de Gran Canaria [tesis doctoral]. Universidad de las palmas de Gran Canaria, departamento de economía y dirección de empresas; 2003.

6. Manera C, Garau J. Los costes de la insularidad en el desarrollo económico. Un contraste en el mediterráneo occidental (1987-2002). Mediterráneo e Historia Económica. 2005; (7): 247 - 268. 
7. Karvelis S, Gioti O. Potential for integrated developmental approach of a small island economy: The case of Milos. Atenas: Panteion University. 2007.

8. Romer P. Increasing Returns and Long-Run Growth. Universidad de Chicago: The Journal of Political Economy. 1986; 94(5): 10021037.

9. Becker G. Human Capital: A Theoretical and Empirical Analysis, with Special Reference to Education. 2da edición. Universidad de Chicago. 1964.

10. Mankiw G. Macroeconomía. 3a Ed. Antoni Bosch editor, Barcelona. 1997.

11. Mesa D, García A, Roa M. Estructura salarial y segmentación en el mercado laboral de Colombia: un análisis de las siete principales ciudades, 2001-2005. Universidad del Rosario: serie documentos de trabajo. 2008; (52).

12. Piore M. Labor Market Segmentation: To What Paradigm Does It Belong?. The American Economic Review. 1983; 73(2): 249-253.

13. Doeringer P. Internal Labor Markets and Noncompeting Groups. The American Economic Review. 1986: 76(2); 48-52.

14. Tenjo J, Bernat L, Uribe Á. Algunos aspectos del funcionamiento del mercado laboral en el sector rural. Departamento Nacional de Planeación Bogotá. 2007.

15. López P, Miguélez Faustino, Lope A, Coller X. La segmentación laboral: hacia una tipología del ámbito productivo. España: Universitat Autònoma de Barcelona y Universidad de Yale. Departmento de Sociología. 1998.

16. Dickens W, Lang K. Testing Dual Labor Market Theory: A Reconsideration Of The Evidence. Cambridge: Working Paper. 1985: 1670.

17. Edwards R, Gordon D, Reich M. Labor Market Segmentation. Lexington (Massachusets): Lexington Books. 1975.

18. Heckman J. Sample Selection Bias as a Specification Error. Econometrica. 1979; 47 (1): 153-161.

\section{BIBLIOGRAFÍA RECOMENDADA}

Uribe J, Ortiz C, García G. La segmentación en el mercado laboral colombiano en la década de los noventa. Universidad del Valle: revista economía institucional. 2007; 9 (16): 189 - 221. 